\title{
Non Lupus Full House Nephropathy - A Clinical and Pathological Correlation Study
}

\author{
Dr Gomathy $\mathrm{S}^{1}$,Dr Usha Samuel ${ }^{2}$,Dr Arun K A ${ }^{3}$,Dr Pradeep B ${ }^{4}$ \\ "Additional Professor, Dept Of Nephrology, Govt.T.D. Medical College, Alappuzha, India ) \\ 2(Professor Dept Of Nephrology, Govt.T.D. Medical College, Alappuzha, India) \\ ${ }_{3}^{3}$ (Senior Resident Dept Of Nephrology, Govt.T .D. Medical College, Alappuzha, India) \\ ${ }^{4}$ (Senior Resident Dept Of Nephrology, Govt.T .D. Medical College, Alappuzha, India)
}

\begin{abstract}
Lupus nephritis is one among the most disabling complications of systemic lupus erythematosus (SLE) as it significantly increases the mortality and morbidity and relentlessly progress to end stage renal disease if not detected and treated promptly. Atypical presentations are well known in SLE and a subset of patients presents with histological evidence of lupus nephritis without other clinical or serological evidence of SLE. Here in this study we analysed the baseline characteristics of 18 such patients with their outcome.

Methodology: 18 patients with biopsy proven non lupus full house nephropathy detected after 2012 were followed up till 2017. Their initial clinical, biochemical and histological characteristics were compared with their outcome.

Results: 2 patients developed ESRD on follow up and 2 became antibody positive .Low serum C3complement, more globally sclerotic glomeruli and crescents showed statistically significant association with progression to ESRD.

Conclusion: The study provides great insight into the details of presentations, biochemical and histological characteristics of non lupus full house nephropathy. The risk factors of faster progression of the disease and evolution of auto antibodies are analysed. Still the true biological nature of this entity is elusive and we recommend more research in this area.
\end{abstract}

Keywords: lupus nephritis, systemic lupus erythematosus, end stage renal disease, non lupus full house syndrome.

\section{Introduction}

Systemic Lupus Erythematosus (SLE) is a prototypical autoimmune disorder which can affect almost all organ systems in the body. The characteristic feature of SLE is a battery of auto antibodies targeted against various cytoplasmic and nuclear antigens which play an important role in the pathogenesis. The detection and characterisation of these antibodies are widely used in the diagnosis of SLE. However diagnostic dilemma can occur when the clinical and serological evidence do not go in support of each other or isolated involvement of a single organ system occur without other evidence of SLE. In this study patients with histological features of lupus nephrits without any clinical or serological evidence of SLE are included. Similar cases were reported from other parts of the world but so far there is no consensus of opinion regarding the treatment or prognosis. Most of the available literature are in the form of case series involving few number of patients.

SLE is an autoimmune disorder with heterogenous manifestations which at times creates confusion in diagnosis and management. The characteristic finding on the skin, joints, nervous system, kidney etc. along with identification of autoantibodies in serology leads to a diagnosis in majority of the cases. Most common non specific complaints are fever, arthralgia and fatigue. The disease commonly affects females of child bearing age with a male to female ratio of $1: 9^{(1)}$. The prevalence ranges from 20 to 150 cases per 100,000 population, with the highest prevalence reported in Brazil, and appears to be increasing as the disease is being recognized more readily with sensitive assays, better clinical awareness and improved survival ${ }^{(2)}$. Asian population is at high risk of SLE and one study documented a point prevalence of 3.2 per 100000 population in northern India ${ }^{(3)}$.

The American College of Rheumatology (ACR) criteria, first published in 1982 and revised in 1997, was used for the classification of SLE .Four out of the 11 criteria have to be fulfilled for a diagnosis of SLE ${ }^{(4)}$. Systemic Lupus International Collaborating Clinics (SLICC) group developed a new set of classification criteria in 2012 which requires both clinical and immunological criteria to classify SLE $^{(5)}$. One important fact which the treating clinician should be aware of is that both these criteria are classification criteria which is more useful for research and are not diagnostic criteria by themselves. Obviously, small subsets of patients with SLE have been reported with negative ANA and anti-dsDNA antibody.

Renal involvement in SLE is still one of the strongest predictor of morbidity and mortality. Lupus nephritis is very common in which autoantibodies form immune complexes and mediate glomerular injury. In some aggressive cases such as class IV LN, up to $25 \%$ of LN patients develop end-stage renal disease (ESRD) 
10 years after onset of renal disease ${ }^{(6)}$. Prompt diagnosis and timely intervention is important to retard the progression of the disease. Because of early recognition and the availability of effective treatment there is marked improvement in 5 year survival from 44 to $95 \%$ in the past 50 years $^{(7)}$.

Literature review shows that a subgroup of patients with typical lupus nephritis proven by biopsy exist without any evidence of clinical or immunological features of SLE. These patients are at risk of delayed diagnosis and may progress to ESRD before getting adequate treatment. The two main types of renal injury identified on renal pathology are immune complex deposition disease as characterized by the known classifications of LN and non-immune complex disease, including thrombotic microangiopathy, podocytopathy and tubulointerstitial disease. Immunofluorescence shows characteristically immunoglobulins viz. $\operatorname{IgG}$, IgA, $\mathrm{IgM}$ and complement components $(\mathrm{C} 3, \mathrm{C} 4, \mathrm{C} 1 \mathrm{q})^{(8)}$. A renal biopsy is mandatory in arriving at the diagnosis because pattern of lupus nephritis on biopsy affects treatment and outcome. Renal biopsy is not specific for the diagnosis of lupus but certin histological features are described which strongly suggests the diagnosis of lupus. They are

- 'Full-house' immunofluorescence staining for IgG, IgM, IgA, C3 and C1

- Extraglomerular immune deposits

- Combined mesangial, subendothelial and subepithelial immune deposits

- The presence of endothelial tubuloreticular inclusions ${ }^{(9)}$

The available knowledge about this renal limited lupus is from few case series in the literature. On follow up some of these patients developed other features of SLE including anti nuclear antibodies. The response to immunosuppressive therapy was also variable.

\section{Aims And Objectives}

1. To study the clinical profile, histology and outcome of patients with non lupus full house nephropathy.

2. To correlate the clinical and histological features with the final outcome .

\section{Materials And Methods}

The study was conducted in the Department of Nephrology, Govt. T D Medical college ,Alappuzha, a tertiary care hospital in Central Kerala ,India. All patients with a renal biopsy diagnosis of non lupus full house from 2012 to 2017 were enrolled into the study. Serum creatinine and 24 hour urine protein estimation was done at initial presentation along with serum C3 complement ,ANA and ANA profile. History regarding features of SLE were collected at the time of enrolment into the study. All biopsy were done as percutaneous procedure with biopsy gun under USG guidance. Two cores of tissues were taken and immediately subjected to ligh microscopy and immunlfluorescence. Patients received appropriate treatment based on clinical, biochemical and histological parameters. They had regular follow up in the OPD every 8 weeks with monitoring of renal function during each visit. Antibodies for SLE were done yearly. Development of clinical features of SLE was also meticulously assessed during each visit. The study was approved by the institutional research committee and ethics committee.

\subsection{Inclusion Criteria}

1. All consecutive patients with biopsy proven non lupus full house nephropathy from 2012 onwards under regular follow up were included as study subjects.

\subsection{Exclusion Criteria}

1. Patients with diabetes, hypertension or any other known risk factor for chronic kidney disease.

2. Patients who did not take proper treatment.

3. Patients who were not on regular follow up.

4. Patients with clinical features or serological evidence of SLE at presentation.

\section{Statistics}

Data analysis was done using SPSS software. Values are expressed as mean plus or minus standard deviations and as percentage for categorical parameters. Differences between groups were compared with Student's t-test for parametric continuous variables. Chi-square test was applied for estimating the occurrence of categorical variables. A $\mathrm{P}$ value $<0.05$ will be used as the threshold of statistical significance.

\section{V . Results}

Eighteen patients satisfying the inclusion criteria were included in the study. They were under constant follow up for variable periods. Age of the participants ranged from 10 years to 62 years with a mean of $39.8 \pm$ 14.4 years. Of the 18 patients there were 6 males (33.3\%) and 12 females (66.7\%). Most common presentation 
was acute glomerulonephritis(AGN) with transient renal failure.Three patients had nephritic syndrome at presentation without significant renal dysfunction and only one patient has persistent proteinuria. One patient presented with features of nephritic nephrotic syndrome .Four patients presented with features of rapidly progressive renal failure including fluid overload and uremic symptoms and required few sessions of hemodialysis initially before biopsy.. Some of the important baseline characteristics are summarised in Table 1 .

Table 1. Baseline clinical and laboratory parameteres

\begin{tabular}{|c|c|c|c|c|c|c|}
\hline S1.No & $\begin{array}{l}\text { Age } \\
\text { (Years) }\end{array}$ & Sex & $\begin{array}{l}\text { Proteinuria } \\
\text { (G/24hr) }\end{array}$ & $\begin{array}{l}\text { Serum } \\
\text { Creatinine } \\
(\mathrm{Mg} / \mathrm{Dl})\end{array}$ & $\begin{array}{ll}\text { Serum } & \text { C3 } \\
\text { Level } & \\
\end{array}$ & $\begin{array}{l}\text { Lupus } \\
\text { Antibodie } \\
\text { S }\end{array}$ \\
\hline 1 & 56 & Female & 5.2 & 3.5 & Normal & Negative \\
\hline 2 & 45 & Male & 2.25 & 5 & Low & Negative \\
\hline 3 & 19 & Male & 0.96 & 4.1 & Normal & Negative \\
\hline 4 & 50 & Female & 6.8 & 1.3 & Normal & Negative \\
\hline 5 & 62 & Female & 1.31 & 2.5 & Low & Negative \\
\hline 6 & 39 & Female & 1.19 & 2.7 & Low & Negative \\
\hline 7 & 38 & Male & 1.5 & 3 & Low & Negative \\
\hline 8 & 55 & Female & 2.18 & 4.5 & Normal & Negative \\
\hline 9 & 31 & Female & 2.8 & 1.3 & Low & Negative \\
\hline 10 & 10 & Female & 7 & 0.6 & Normal & Negative \\
\hline 11 & 20 & Female & 1.4 & 1.3 & Normal & Negative \\
\hline 12 & 31 & Female & 3 & 1.8 & Normal & Negative \\
\hline 13 & 32 & Female & 3.6 & 1 & Normal & Negative \\
\hline 14 & 33 & Female & 2.6 & 0.9 & Low & Negative \\
\hline 15 & 56 & Female & 2.49 & 4 & Normal & Negative \\
\hline 16 & 48 & Male & 1.3 & 1.1 & Normal & Negative \\
\hline 17 & 41 & Male & 3.6 & 1.9 & Low & Negative \\
\hline 18 & 51 & Male & 0.3 & 2.6 & Low & Negative \\
\hline
\end{tabular}

The clinical presentation of the patients are summarized in Table2

Table 2.

\begin{tabular}{|c|c|c|c|}
\hline S1 No & Clinical presentation & Serum Creatinine & Follow up \\
\hline 1 & AGN & 3.5 & Resolved \\
\hline 2 & RPGN & 5 & ESRD \\
\hline 3 & RPGN & 4.1 & ESRD -Died \\
\hline 4 & Nephrotic syndrome & 1.3 & Resolved \\
\hline 5 & AGN & 2.5 & Resolved \\
\hline 6 & AGN & 2.7 & Resolved \\
\hline 7 & AGN & 3 & Resolved \\
\hline 8 & RPGN & 4.5 & Resolved \\
\hline 9 & AGN (DPGN) & 1.3 & Resolved \\
\hline 10 & Nephrotic syndrome & 0.6 & Persistent \\
\hline 11 & Nephrotic syndrome & 1.3 & Resolved \\
\hline 12 & $\mathrm{AGN}(\mathrm{DPGN})$ & 1.8 & Resolved \\
\hline 13 & AGN & 1 & Resolved \\
\hline 14 & AGN & 0.9 & Resolved \\
\hline 15 & RPGN & 4 & Resolved \\
\hline 16 & AGN & 1.1 & Resolved \\
\hline 17 & AGN & 1.9 & Resolved \\
\hline 18 & Nephritic nephriotic & 2.6 & Resolved \\
\hline
\end{tabular}


All participants were subjected to renal biopsy and sample was processed for light microscopy and immunofluorescence. Presence of crescents, degree of glomerulosclerosis and immunofluorescence staining for IgG, IgA, IgM, C3 complement, $\kappa$ and $\lambda$ light chains ,C1q complement was done. Table. 3 summarises the renal biopsy results of patients.

Table 3 . Biopsy findings of the patients -light microscopy and immunofluorescence (IF) *globally sclerotic glomeruli

\begin{tabular}{|c|c|c|c|c|c|c|c|c|c|c|}
\hline S1 No & $\begin{array}{l}\text { GSG } \\
*(\%) \\
\end{array}$ & $\begin{array}{l}\text { Crescent } \\
\mathrm{s}(\%)\end{array}$ & $\begin{array}{l}\text { TIAtroph } \\
\mathrm{y} * *(\%)\end{array}$ & $\begin{array}{l}\mathrm{IgG} \\
\text { (IF) }\end{array}$ & $\begin{array}{l}\text { IgA } \\
\text { (IF) }\end{array}$ & $\operatorname{IgM}(\mathrm{IF})$ & C3 (IF) & $\mathrm{C} 1 \mathrm{q}(\mathrm{IF})$ & $\kappa(\mathrm{IF})$ & $\lambda(\mathrm{IF})$ \\
\hline 1 & 5 & Absent & 0 & $1+$ & $1+$ & $2+$ & $1+$ & Absent & $\begin{array}{l}\text { Abse } \\
\text { nt }\end{array}$ & $\begin{array}{l}\text { Absen } \\
\mathrm{t}\end{array}$ \\
\hline 2 & 22.2 & 72.2 & 10 & $2+$ & $2+$ & $2+$ & $2+$ & $3+$ & $2+$ & $3+$ \\
\hline 3 & 50 & 25 & 40 & $3+$ & $1+$ & $1+$ & $1+$ & $3+$ & $1+$ & $3+$ \\
\hline 4 & 11.7 & Absent & 10 & $1+$ & $1+$ & Absent & $2+$ & $2+$ & $1+$ & $1+$ \\
\hline 5 & 22.2 & Absent & 10 & $3+$ & Absent & $3+$ & $4+$ & $2+$ & $2+$ & $3+$ \\
\hline 6 & 6.25 & Absent & 0 & $2+$ & $2+$ & $1+$ & $3+$ & Absent & $2+$ & $2+$ \\
\hline 7 & 0 & Absent & 0 & $2+$ & Absent & Absent & $3+$ & $3+$ & $3+$ & $1+$ \\
\hline 8 & 17.9 & Absent & 25 & $2+$ & Absent & $1+$ & $2+$ & $2+$ & $2+$ & $2+$ \\
\hline 9 & 11.7 & Absent & 5 & $3+$ & Absent & $3+$ & $3+$ & $3+$ & $1+$ & $1+$ \\
\hline 10 & 0 & Absent & 0 & $3+$ & $1+$ & $1+$ & $3+$ & $2+$ & $1+$ & $2+$ \\
\hline 11 & 21 & Absent & 20 & $3+$ & Absent & $1+$ & $1+$ & $1+$ & $2+$ & $1+$ \\
\hline 12 & 11.7 & Absent & 5 & $3+$ & Absent & $3+$ & $3+$ & $3+$ & $3+$ & $3+$ \\
\hline 13 & 0 & Absent & 0 & $3+$ & $2+$ & $3+$ & $3+$ & $3+$ & $1+$ & $3+$ \\
\hline 14 & 0 & Absent & 0 & $3+$ & $1+$ & $2+$ & $3+$ & $3+$ & $\begin{array}{l}\text { Abse } \\
\text { nt }\end{array}$ & $1+$ \\
\hline 15 & 0 & Absent & 5 & $3+$ & $2+$ & $3+$ & $3+$ & $2+$ & $1+$ & $2+$ \\
\hline 16 & 0 & Absent & 0 & $3+$ & $3+$ & $2+$ & $3+$ & $1+$ & $1+$ & $1+$ \\
\hline 17 & 0 & Absent & 0 & $3+$ & $1+$ & $1+$ & $3+$ & $3+$ & $2+$ & $1+$ \\
\hline 18 & 20 & 20 & 40 & $3+$ & $2+$ & Absent & $3+$ & $3+$ & $3+$ & $3+$ \\
\hline
\end{tabular}

All patients received treatment from our hospital. Immunosuppression was given based on the clinical picture as there are no standard guidelines available at present regarding treatment. The major outcomes considered at the time of analysis are progression to end stage renal disease and development of autoantibodies. The mean duration of follow up was 2.3 years. Two patients reached the end stage renal disease despite getting adequate immunosuppression. Auto antibodies became positive in two patients on follow up.

Details of follow up are given in Table.4

Table 4. Follow up details of the patients

\begin{tabular}{|c|c|c|c|}
\hline Sl No & $\begin{array}{l}\text { Duration of follow up in } \\
\text { years }\end{array}$ & $\begin{array}{lll}\text { End } & \text { stage } & \text { renal } \\
\text { disease } & & \\
\end{array}$ & Auto antibodies \\
\hline 1 & 4 & No & No \\
\hline 2 & 3 & Yes & No \\
\hline 3 & 1.5 & Yes & No \\
\hline 4 & 3 & No & No \\
\hline 5 & 4 & No & No \\
\hline 6 & 4.5 & No & No \\
\hline 7 & 1.5 & No & No \\
\hline 8 & 2 & No & No \\
\hline 9 & 0.5 & No & No \\
\hline 10 & 2 & No & $\begin{array}{l}\text { Yes (ANA, Anti } \\
\text { dsDNA,Anti TPO) }\end{array}$ \\
\hline 11 & 2 & No & No \\
\hline 12 & 1.5 & No & No \\
\hline 13 & 4 & No & No \\
\hline
\end{tabular}




\begin{tabular}{|c|c|c|c|}
\hline 14 & 1 & No & No \\
\hline 15 & 2 & No & No \\
\hline 16 & 2.5 & No & No \\
\hline 17 & 3 & No & No \\
\hline 18 & 1 & No & $\begin{array}{l}\text { Yes (ANA, Anti } \\
\text { dsDNA) }\end{array}$ \\
\hline
\end{tabular}

Patient 2 developed end stage renal disease 3 years after the biopsy and is now on maintenance hemodialyisis awaiting transplantation. He presented at the age of 45 years with hypertension and renal failure with a serum creatinine of $5 \mathrm{mg} / \mathrm{dl}$ and protenuria of $2.25 \mathrm{~g} / \mathrm{day}$. ANA and Anti dsDNA was negative . Serum C3 complement level was low. Renal biopsy showed $72 \%$ crescents (12 cellular and 1 fibrocellular) ,22\% obsolescent glomeruli and $10 \%$ tubulointestitial fibrosis. Immunofluorecence showed full house pattern. Despite timely intervention his disease progressed to end stage renal disease.

Patent 3 was a 19 year old male who presented with rapidly progressive renal failure with accelerated hypertension. Initial serum creatinine was $4.1 \mathrm{mg} / \mathrm{dl}$ with $966 \mathrm{mg} / \mathrm{day}$ proteinuria. ANA ,Anti dsDNA was negative and serum C3 complement was normal. Biopsy revealed 50\% globally sclerotic glomeruli with $25 \%$ crescents (1 cellular and 3 fibrocellular) and 40\% tubular atrophy. Immunofluorecence showed full house pattern. Initially managed conservatively but the course was complicated by 2 episodes of acute worsening associated with acute diarrhoeal disease and respiratory infection which required dialysis. Rapidly progressed to end stage renal failure by one and a half year and maintenance hemodialysis was initiated . He expired after few weeks due to infective endocarditis. Both these patients were checked for autoantibodies during follow up but was negative.

Patient 10 ,was a girl presented at the age of 10 years with nephrotic proteinuria without renal failure. She also had hypertension at the initial presentation and proteinuria was steroid resistant. ANA, Anti dsDNA were negative. C3 complement was normal. Serology for Hep B, C and HIV was negative. Biopsy is suggestive of membranous nephropathy and showed no obsolescent glomeruli or crescents. Tubulointestitum was unremarkable. IF showed full house pattern. She received Tacrolimus, mycophenolate mofetil each in succession in combination with steroids (oral prednisolone) and as she remained nonresponsive she was also given four doses of Rituximab (500mg weekly once for four weeks ) and is now under followup . At two and a half months of RTX now proteinuria has decreased by 50\%. After 2 years she became positive for ANA, Anti dsDNA .Repeat serum C3 was low. Subsequently she also developed autoimmune thyroiditis with TSH >100 IU and anti Thyroid peroxidise (Anti TPO>1000).

Patient 18 was 51 year old male was referred to our hospital for evaluation of renal failure (serum creatinine $2.6 \mathrm{mg} / \mathrm{dl}$ ). He also had hypertension and 24 hour urine protein was $300 \mathrm{mg}$. Urine microscopy showed plenty of RBCs. Renal biopsy revealed glomerular basement membrane thickening ,mesangial matrix proliferation, focal endocapillary proliferation, neutrophilic exudate $20 \%$ obsolescent glomeruli and one crescent. There was $40 \%$ tubular atrophy with intestitial lympho plasmacytic infiltrate. He became positive for ANA and Anti dsDNA after one year of diagnosis though he did not develop any clinical features of SLE. At present he is on prednisolone and cyclophosphamide and renal function is stable.

The biochemical and histological parameters were analyzed to study their effect on the outcome.

\section{V.1Proteinuria}

Patients had proteinuria ranging from $300 \mathrm{mg}$ to $7 \mathrm{~g}$ per day with a median of $2.3 \mathrm{~g} /$ day. $27.8 \%$ had nephrotic range proteinuria. There was no significant difference between males and females with regard to proteinuria (p value 0.184). Similarly presence of crescents, severity of glomerular sclerosis, low serum C3 or $\mathrm{C} 1 \mathrm{q}$ in IF did not show any significant difference in patients presented with nephrotic syndrome. Further on following up these patients we could not demonstrate that prevalence of higher grades of proteinuria at presentation are more common either in patients who became antibody positive or in patients who progressed to end stage renal disease.

\section{V.2 Serum creatinine}

The mean serum creatinine at presentation was $2.3 \pm 1.3 \mathrm{mg} / \mathrm{dl}$. The values ranged from $0.6 \mathrm{mg} / \mathrm{dl}$ to 5 $\mathrm{mg} / \mathrm{dl}$. The mean creatinine in males was 2.9 and that in females was $2.1 \mathrm{mg} / \mathrm{dl}$ but this difference was not statistically significant(pvalue 0.955). The renal failure was more severe among patients with crescents in their biopsy (mean serum creatinine $3.9 \mathrm{vs} 2 \mathrm{mg} / \mathrm{dl}$,p value 0.034 ). Auto antibody positivity or end stage renal disease were not found to be significantly more common among patients with high serum creatinine at presentation. 


\section{V.3 Serum C3 complement}

$44 \%(8 / 18)$ presented with low serum C3 at the time of diagnosis. Patients with low C3 had higher mean age (42.5 yrs) compared to patients with normal C3 (37.7 years) with a significant Pvalue of 0.021. Males and females did not show significant difference in C3 level. Low C3 was more frequently noted in patients with auto antibodies and progression to end stage renal disease.( $p$ value 0.028). But such a difference was not seen in patients with crescents or severe glomeruloscleois.

\section{V.4 Globally sclerotic glomeruli}

Based on the severity sclerosis patients were divided into groups as give in Table 4 Table 4. GSG

\begin{tabular}{|l|l|}
\hline GSG (\%) & Frequency \\
\hline Nil & 6 \\
\hline$<10$ & 3 \\
\hline $10-19$ & 4 \\
\hline $20-29$ & 4 \\
\hline $50-69$ & 1 \\
\hline
\end{tabular}

$66.7 \%$ had atleast one globally sclerotic glomeruli. No statistically significant difference observed among different age groups or sex with GSG. More number of GSG was found to be more frequently associated with crescents in biopsy(pvalue 0.029) and C1q on immunofluorecence ( $\mathrm{p}$ value 0.024) and similar is the case with end stage renal disease.(p value 0.034 ).

\section{V.5 Crescents}

Only three patients had crescents on renal biopsy. 2 of them progressed to stage $\mathrm{V}$ chronic kidney disease and one became antibody positive on follow up. All the three patients were males.

\section{V.6 C1q staining}

$88 \%$ patients were C1q positive on IF. No age or sex difference in C1q positivity was observed. Similar is the case with high serum creatinine at presentation. Statistically significant association was seen with number of GSG but not with progression to end stage renal disease or antibody positivity. ESRD

Low serum C3, more GSG and crescents showed statistically significant association with progression to

\section{Discussion}

In this study we tried to elucidate the clinical and histological pattern of non lupus full house nephropathy and made an attempt to study their natural course and progression. A definite pattern of their biological behaviour could not be drawn from the available literature mainly because of the scarcity of clinical data.

The mean age of patients in our cohort was 39.8 years consistent with the observation that SLE is a disease of the reproductive age group. Of these two third were females. It is known that males are more prone to lupus nephritis especially severe forms including class IV lupus nephritis and male patients has a poor renal outcome $^{(10)}$. In this study also male patients formed one third of the cases and two of the six male patients progressed to end stage renal disease. The presence of low serum C3 complement, higher grades of glomerulosclerosis and crescents were found to be associated with the progression to ESRD.

The remaining 16 patients did not show any evidence of progressive renal failure even after relatively long follow up. Electron microscopy to look for tubule reticular inclusions is useful in this setting.We could not use EM because of financial constraints. According to Andrew Sharman, the term C1q nephropathy should be preferred when these histological features are seen in the absence of overt lupus, when C1q deposition is dominant and when tubuloreticular bodies are absent. In his report of 9 patients initially considered as lupus nephritis none of them progressed to overt lupus and the treatment response to immunosuppression was very poor $^{(11)}$.

C1q nephropathy was first described by Jennette and Hipp in $1985^{(12) .}$ It is believed that it is an immune complexes mediated glomerular disease. The diagnostic criteria laid down were:

1. Dominant staining for $\mathrm{C} 1 \mathrm{q}$

2. Mesangial deposits by electron microscopy

3. Absence of clinical or serology of SLE 
C1q nephropathy presents as full house nephropathy closely resembling lupus nephritis histologically. But the clinical course is entirely different. C1q nephropathy does not progress to SLE and show no response to immunosuppression. The presence of tubuloreticular inclusions due to high quantity of interferon alpha in lupus nephrits is a feature which helps to distinguish it from C1q nephropathy ${ }^{(13)}$. Full-house nephropathy can also be seen in clinical conditions such as posthepatic cirrhosis, diabetic nephropathy, membranous nephropathy, membranoproliferative glomerulonephritis. Two patients turned out to be antibody positive on follow up. But they were not having other clinical features of SLE. From our analysis of data we were unable to tell the factors which could predict the chance of future development of auto antibodies.

When we look into the published data, there are reports of SLE and lupus nephritis which were not associated with demonstrable autoantibodies at presentation but developed autoantibodies on follow up. Cairns et al in 1979 reported 11 such cases out of which 3 presented with features of lupus nephritis initially without autoantibodies in serum ${ }^{(15)}$. Gianviti et al published a case series in 1999 in which he described 3 patients presented initially with seronegative lupus and receieved immunosuppression. Between 5 and 10 years of follow up all the three patients became ANA and antidsDNA positive ${ }^{(16)}$. Ozdemir and colleagues reported a case of post partum renal failure with biopsy suggestive of lupus nephritis .Patient improved after prompt intervention and became ANA positive on further follow up ${ }^{(17)}$.

In contrast to the above mentioned cases, serology remained negative over a long period of follow up in another subset of patients. Ana Huerta et al described four patients with lupus like nephritis and none of them became seropositive on follow up. The author referred to them as renal limited lupus like nephritis considering their unique presentation and clinical course. All four patients reached the stage of end stage renal disease in spite of receiving aggressive immunosuppressive therapy ${ }^{(9)}$. In this study also the two patients who reached ESRD remained antibody negative. Eventhough a firm conclusion cannot be drawn at this level, the outcome does not appear to be favourable. The prognosis of these patients are worse or atleast as bad as those patients with nephritis who meet the criteria for $\mathrm{SLE}^{(18)}$. Kim et al reported a similar case of nephritis with persistent absence of autoantibodies in serum. But this patient showed good response to treatment even after 2 years of follow up ${ }^{(19)}$.

Five percentage of SLE can present with negative anti nuclear antibodies in serology. Such patients with clinical features of SLE without serological evidence can also be complicated by full house nephropathy (14). There are many causes postulated to account for the absence of antibodies in these patients. They are

1. Technical inaccuracy.

Instead of rat liver substrate which was used earlier for ANA testing the increasing use of human epithelial (HEp-2) substrate has increased the sensitivity of ANA assays as a result perceived incidence of ANA negative SLE has decreased.

2. Low circulating level since antibodies are trapped in immune complexes.

This has been described in five patients with lupus nephritis whose ANAs, which were primarily reactive with DNA, were not detected in the serum by indirect immunofluorescence until the ANAs were dissociated from circulating immune complexes.

3. Urinary loss of antibodies due to heavy proteinuria.

The tests for ANA became positive upon clinical recovery with reduction in proteinuria.

4. Prozone phenomena.

5. Close mimicker of lupus nephritis viz. C1q nephropathy ${ }^{(20)}$.

Immune dysregulation in lupus nephritis depends on various genetic and environmental factors. The variable presentations and clinical course could be largely attributed to the genetic make up of different individuals $^{(21)}$

\section{VII .Conclusions}

According to our observations and previous studies what we could infer is that non lupus full house nephropathy also follows a heterogenous course. This study shows that low serum C3 at presentation, glomerulosclerosis and crescents in biopsy are associated with progressive renal disease. However this statistical associations should be interpreted with caution since the sample size is very small. Only two patients developed autoantibodies on follow up. This also hints at the possibility of other entitites like the so called c1q nephropathy which has to be ruled out in every case.

\section{Suggestions}

As early recognition and prompt treatment is of paramount importance in lupus nephritis, patients with renal limited forms of the disease are likely to evade clinical detection in the initial stages because of the lack of florid manifestations a the usual case. Hence high index of suspicion is warranted and any case of renal failure especially in the lupus prone age group should be actively investigated and followed up. Existing data are scarce 
and grossly insufficient to define the natural course, type of treatment and prognosis of non lupus full house nephropathy. So we recommend further studies on this entity with larger sample size and long term follow up.

\section{References}

[1]. M.E. Soto MV, F. Guillén, J.A. Simón, E Arena, P A Reyes. Gender impact in systemic lupus erythematosus. Clinical and Experimental Rheumatology. 2004;22: 713-721.

[2]. Tsokos GC. Systemic Lupus Erythematosus. New England Journal of Medicine. 2011;365(22):2110-21. PubMed PMID: 22129255 .

[3]. A.N. Malaviya Rrs, Y.N. Singh, S.K. Kapoor And A. Kumar. Prevalence of Systemic Lupus Erythematosus in India. lupus (1993) 2,115-118.

[4]. Hochberg MC. Updating the American college of rheumatology revised criteria for the classification of systemic lupus erythematosus. Arthritis \& Rheumatism. 1997;40(9):1725-

[5]. Petri M, Orbai A-M, Alarcón GS, Gordon C, Merrill JT, Fortin PR, et al. Derivation and Validation of Systemic Lupus International Collaborating Clinics Classification Criteria for Systemic Lupus Erythematosus. Arthritis and rheumatism. 2012;64(8):2677-86. PubMed PMID: PMC3409311.

[6]. Ortega L, DS, OL, VPaGC. Lupus nephritis: pathologic features, epidemiology and a guide to therapeutic decisions. Lupus (2010) $19,557-574$.

[7]. Johnson RJ, Feehally J, Floege J. Comprehensive clinical nephrology: Elsevier Health Sciences; 2014.

[8]. Simmons SC, Smith ML, Chang-Miller A, Keddis MT. Antinuclear Antibody-Negative Lupus Nephritis with Full House Nephropathy: A Case Report and Review of the Literature. American Journal of Nephrology. 2015;42(6):451-9.

[9]. Huerta A, Bomback AS, Liakopoulos V, Palanisamy A, Stokes MB, D'Agati VD, et al. Renal-limited 'lupus-like' nephritis. Nephrology, dialysis, transplantation : official publication of the European Dialysis and Transplant Association - European Renal Association. 2012 Jun;27(6):2337-42. PubMed PMID: 22207326

[10]. J. F. Molina, C. Drenkard, J. Molina et al., "Systemic lupus erythematosus in males: a study of 107 Latin American patients," Medicine, vol. 75, no. 3, pp. 124-13

[11]. Sharman A, Furness P, Feehally J. Distinguishing C1q nephropathy from lupus nephritis. Nephrology Dialysis Transplantation. 2004;19(6):1420-6.0, 199

[12]. Devasahayam J, Erode-Singaravelu G, Bhat Z, Oliver T, Chandran A, Zeng X, et al. C1q Nephropathy: The Unique Underrecognized Pathological Entity. Analytical cellular pathology (Amsterdam)2015:490413. PubMed PMID: PMC4657067.

[13]. Kun C-KSaEW-L. A Young Lady with Full-house Nephropathy and Negative Anti-nuclear Antibody. Hong Kong Bull Rheum Dis 2009;9:23-25.6

[14]. Elcioglu OC, Ozkok A, Bakan A, Tuncan S, Ozluk Y, K1lıcarslan I, et al. A Case of Full-House Nephropathy with Anti-Nuclear Antibody Negative Lupus. Turkish Nephrology Dialysis Transplantation. 2014;23(2):161-3.

[15]. Cairns Sa, Corbet Cl, Mallick Np, Lawler W, Williams G. The delayed appearance of an antinuclear factor and the diagnosis of systemic lupus erythematosus in glomerulonephritis. Postgraduate Medical Journal (October 1979) 55, 723-727. 1979.

[16]. Gianviti A, Barsotti P, Barbera V, Faraggiana T, Rizzoni G. Delayed onset of systemic lupus erythematosus in patients with "fullhouse" nephropathy. Pediatric Nephrology. 1999 1999//;13(8):683-7.

[17]. FN Ozdemir RE, A Akcay, BH Ozdemir, S Sezer, E Kuscu and M Haberal. Seronegative systemic lupus erythematosus: etiology of nephrotic syndrome and acute renal failure in early postpartum period. Lupus (2005) 14, 629-631.

[18]. Bomback AS, Appel GB. Updates on the treatment of lupus nephritis. Journal of the American Society of Nephrology : JASN. 2010 Dec;21(12):2028-35. PubMed PMID: 21051743.

[19]. Park CY, J-sK, SpS, W-JH, YHL, and N, et al. A Case of Renal-limited Lupus-like Nephritis. Soonchunhyang Medical Science 19(2):104-107, December 2013.

[20]. Kim HA, Chung JW, Park HJ, Joe DY, Yim HE, Park HS, et al. An antinuclear antibody-negative patient with lupus nephritis. The Korean journal of internal medicine. 2009 Mar;24(1):76-9. PubMed PMID: 19270487. Pubmed Central PMCID: 2687656.

[21]. Anders H-J, Fogo AB, editors. Immunopathology of lupus nephritis. Seminars in immunopathology; 2014: 144-150. 